\title{
[gw22-e0086] COMPARISON BETWEEN CT ANGIOGRAPHY BASED SCORING SYSTEM AND CORONARY ANGIOGRAPHY BASED SCORING SYSTEM IN PREDICTING MAJOR CORONARY EVENTS
}

Luyue Gai, Bin He, Jingjing Gai, Huaiyu Qiao, Shuoyang Zhang, Zhiwei Guan, Li Yang, Yundai Chen Chinese Pla General Hospital, Beijing, China

10.1136/heartjnl-2011-300867.340

Purpose Acute coronary events often occur suddenly without warning. The mortality and morbidity are high. There has been many scoring system to predict coronary events. However, none is in combination with CT angiography and clinical variables which we think that it might improve the outcomes.

Method The pts who underwent CCTA from January 2008 to February 2010 were included in the study. Any patient who developed acute ST elevated myocardial infarction (STEMI) or non-ST elevated acute myocardial infarction (NSTEMI) or cardiac death after CCTA was considered a major coronary event (MACE). Colour-coded coronary plaque analysis was performed and total plaque score calculated. MASE score was calculated as follow: death 3 points, heart failure 2 points, STEMI 1, NSTEMI 0.5, multiplied by the number of abnormal segments. Post events coronary angiography was performed to determine the clinical Syntax score. CSS=(SYNTAX Score $) \times($ modified ACEF score $)$; Clinical Plaque score $=$ (plaque score $) \times$ (modified ACEF score); Clinical MACE score $=($ MACE score $) \times$ (modified ACEF score).

Results From February 2008 to February 2010 a total of 8557 cases of CCTA were performed our hospital. Among them 19 cases developed MACE during the follow-up. Six scoring systems were compared to select best predictor of MACE. The systems that combined with both CT angiography and clinical variables archived higher score than clinical score alone. Comparison of 7 Risk Scoring Systems Clinical plaque score versus clinical MACE score $\mathrm{R}=0.84<0.01$ clinical MACE score versus CSS $\mathrm{R}=0.89<0.01$ Clinical plaque score versus CSS $\mathrm{R}=0.85<0.01$ Min score versus CSS $\mathrm{R}=0.79<0.01$ Clinical plaque score versus Grace $\mathrm{R}=0.68<0.01$ clinical MACE score versus Grace $R=0.57<0.01$ CSS versus Grace $R=0.65<0.01$ CONCLUSION: The scoring systems that combine with both CT angiography and clinical variables could non-invasively achieve the same degree of precision as the Syntax score in predicating the MACE. 